\title{
Divisors and specializations of Lucas polynomials
}

\author{
Tewodros Amdeberhan, Mahir Bilen Can, and Melanie Jensen
}

\begin{abstract}
Three-term recurrences have infused a stupendous amount of research in a broad spectrum of the sciences, such as orthogonal polynomials (in special functions) and lattice paths (in enumerative combinatorics). Among these are the Lucas polynomials, which have seen a recent true revival. In this paper one of the themes of investigation is the specialization to the Pell and Delannoy numbers. The underpinning motivation comprises primarily of divisibility and symmetry. One of the most remarkable findings is a structural decomposition of the Lucas polynomials into what we term as flat and sharp analogs.
\end{abstract}

AMS 2010 SUBJECT ClassificAtions: 05A10, 11 B39.

KEYWORDS AND PHRASES: Lucas polynomials, flat and sharp lucanomials, divisors, Iwahori-Hecke algebra.

\section{Introduction}

In this paper, we focus on two themes in Lucas polynomials, the first of which has a rather ancient flavor. In mathematics, often, the simplest ideas carry most importance, and hence they live longest. Among all combinatorial sequences, the (misattributed) Pell sequence seem to be particularly resilient. Defined by the simple recurrence

$$
P_{n}=2 P_{n-1}+P_{n-2} \text { for } n \geq 2,
$$

with respect to initial conditions $P_{0}=0, P_{1}=1$, Pell numbers appear in ancient texts (for example, in Shulba Sutra, approximately 800 BC). The first eight values of $P_{n}$ are given by $(0,1,2,5,12,29,70,169)$, and the remainders modulo 3 are

$$
\left(P_{0}, P_{1}, P_{2}, P_{3}, P_{4}, P_{5}, P_{6}, P_{7}\right) \equiv_{3}(0,1,2,2,0,2,1,1) .
$$

It is hard not to appreciate (1.2), since the sequence $\left(P_{n} \bmod 3: n \geq 0\right)$ is periodic, of period 8 . This fact is easily proven by inducting on $n$, and by using the recurrence from (1.1).

arXiv: 1406.0432 
Let $m, n$ be two positive integers, and let $p$ be a prime number. By the Fundamental Theorem of Arithmetic, there exists a unique expression of the form $m / n=p_{1}^{f_{1}} \cdots p_{r}^{f_{r}}$ for some integers $f_{i} \in \mathbb{Z}$, and prime numbers $p_{i}$. The $p$-adic valuation of $m / n$ is then defined by

$$
\nu_{p}\left(\frac{m}{n}\right)= \begin{cases}f_{i} & \text { if } p=p_{i}, \\ 0 & \text { otherwise }\end{cases}
$$

Although the ancients did not document their $p$-adic arithmetic, it is fair to assume that the tools for proving the following interesting consequence of the 8-periodicity were at their disposal: the 3 -adic valuation of the Pell sequence is of the form

$$
\nu_{3}\left(P_{n}\right)= \begin{cases}\nu_{3}(3 k) & \text { if } n=4 k, \\ 0 & \text { otherwise }\end{cases}
$$

Indeed, the case $4 \nmid n$ is evident from the periodicity and (1.2). For the other cases, we use the following well-known consequence

$$
P_{m+n}=P_{m} P_{n+1}+P_{m-1} P_{n}
$$

of the recurrence (1.1).

Suppose $n=4(3 k+1)$. From (1.4), $P_{12 k+4}=P_{12 k} P_{5}+P_{12 k-1} P_{4}$. By induction, $\nu_{3}\left(P_{4(3 k)}\right)=2+\nu_{3}(k), \nu_{3}\left(P_{12 k-1}\right)=0$. By direct calculation $\nu_{3}\left(P_{5}\right)=0, \nu_{3}\left(P_{4}\right)=1$. So,

$$
\nu_{3}\left(P_{n}\right)=1=\nu_{3}(4(3 k+1)) .
$$

Suppose $n=4(3 k+2)$. From (1.4), $P_{12 k+8}=P_{12 k} P_{9}+P_{12 k-1} P_{8}$. By induction, $\nu_{3}\left(P_{4(3 k)}\right)=2+\nu_{3}(k), \nu_{3}\left(P_{12 k-1}\right)=0$. By direct calculation $\nu_{3}\left(P_{9}\right)=0, \nu_{3}\left(P_{8}\right)=1$. So,

$$
\nu_{3}\left(P_{n}\right)=1=\nu_{3}(4(3 k+2)) .
$$

Suppose $n=4(3 k+3)$. Once more, apply (1.4) repeatedly to obtain

$$
\begin{aligned}
P_{12 k+12} & =P_{8 k+8} P_{4 k+5}+P_{8 k+7} P_{4 k+4} \\
& =\left(P_{4 k+4} P_{4 k+5}+P_{4 k+3} P_{4 k+4}\right) P_{4 k+5}+P_{8 k+7} P_{4 k+4} \\
& =P_{4 k+4}\left[\left(P_{4 k+5}+P_{4 k+3}\right) P_{4 k+5}+P_{8 k+7}\right] \\
& =P_{4 k+4}\left[\left(P_{4 k+5}+P_{4 k+3}\right) P_{4 k+5}+P_{4 k+3} P_{4 k+5}+P_{4 k+2} P_{4 k+4}\right] \\
& =P_{4 k+4}\left[2\left(P_{4 k+4}+P_{4 k+3}\right) P_{4 k+5}+P_{4 k+3} P_{4 k+5}+P_{4 k+2} P_{4 k+4}\right] \\
& =P_{4 k+4}\left[\left(2 P_{4 k+5}+P_{4 k+2}\right) P_{4 k+4}+3 P_{4 k+3} P_{4 k+5}\right] .
\end{aligned}
$$


Since $\nu_{3}\left(3 P_{4 k+3} P_{4 k+5}\right)=1, \nu_{3}\left(P_{4 k+4}\right)=1+\nu_{3}(k+1)$ and $3 \mid\left(2 P_{4 k+5}+\right.$ $\left.P_{4 k+2}\right)$, it follows that the terms in $\left[\left(2 P_{4 k+5}+P_{4 k+2}\right) P_{4 k+4}+3 P_{4 k+3} P_{4 k+5}\right]$ are divisible by exactly 3 . Combining these facts,

$$
\nu_{3}\left(P_{n}\right)=\nu_{3}\left(P_{4 k+4}\right)+1=\nu_{3}(3(k+1))+1=\nu_{3}(4(3 k+3)) .
$$

The proof of (1.3) is complete.

We denote by $\mathbb{N}$ the set of all non-negative integers, and by $\mathbb{P}$ the set of positive integers.

Corollary 1.5. Given $k \in \mathbb{P}$, let $n=4 k$. Then $P_{n}^{2}$ does not divide $P_{n^{2}}$.

Proof. A simple use of (1.3) leads to $\nu_{3}\left(P_{n^{2}}\right)=1+2 \nu_{3}(k)<\nu\left(P_{n}^{2}\right)=$ $2+2 \nu_{3}(k)$.

The hypothesis of Corollary 1.5 is restrictive in the sense that $n$ is assumed to be a multiple of 4 . Our effort to remove the restriction has led us to consider the same question in a more general context, for a family of polynomials $L_{n}=L_{n}(s, t) \in \mathbb{N}[s, t]$, known as Lucas polynomials, ${ }^{1}$ defined by

$L_{n}=s L_{n-1}+t L_{n-2}, \quad$ subject to the initial conditions $L_{1}=1, L_{0}=0$.

Obviously, when $s=2, t=1$ we recover Pell numbers. At the same time, Lucas polynomials have many other interesting specializations:

1. $L_{n}(1,1)=f_{n}, n$-th Fibonacci number;

2. $L_{n}(2,-1)=n$, for all $n \geq 0$;

3. $L_{n}(s, 0)=s^{n-1}$, for all $n \geq 1$;

4. $L_{2 n}(0, t)=0$, and $L_{2 n+1}(0, t)=t^{n}$, for all $n \geq 0$;

5. $L_{n}(q+1,-q)=1+q+\cdots+q^{n-1}$, the standard $q$-analog of $n$.

The main result that motivated our paper is the following truly remarkable multiplicity-free property of Lucas polynomials:

Theorem 1.6. Let $d \neq 1$ be a divisor of $n \in \mathbb{P}$. Then $L_{d}^{2}$ does not divide $L_{n}$.

Note that, by evaluating $L_{n}$ at $s=2, t=1$, we obtain Corollary 1.5 without any restriction on $n$.

\footnotetext{
${ }^{1} \operatorname{In}[9], L_{n}$ is denoted by $\{n\}$.
} 
From an algebraic point of view, "binomial coefficients" are the special values of the $\mathbb{Q}$-valued function

$$
\left(\begin{array}{l}
x_{n} \\
x_{k}
\end{array}\right)=\frac{x_{n} x_{n-1} \cdots x_{n-k+1}}{x_{k} x_{k-1} \cdots x_{2} x_{1}}, \quad(\text { when } 1 \leq k \leq n)
$$

defined on a sequence $\left(x_{i}\right)_{i \in \mathbb{P}}$ of non-negative integers $x_{i}$. For an arbitrary integer sequence, the binomials in (1.7) need not be integral. However, it follows from well-known combinatorial reasons that for the sequence $x_{i}=i$, for all $i \in \mathbb{N}$, the binomial coefficients are integers. When $x_{n}$ is the $n$ th Fibonacci number, the associated binomial-like coefficients, customarily called fibonomials, are integers as well.

In general, to understand the nature of integer sequences, it is often helpful to study them by introducing extra parameters. For Fibonacci numbers there are many polynomial generalizations, and the family of Lucas polynomials is one of them. In analogy, the Lucas polynomial analog of the fibonomials are defined by

$$
\left(\begin{array}{c}
L_{n} \\
L_{k}
\end{array}\right):=\frac{L_{n} L_{n-1} \cdots L_{n-k+1}}{L_{k} L_{k-1} \cdots L_{1}} .
$$

The tapestry

$$
\left(\begin{array}{c}
L_{m+n} \\
L_{m}
\end{array}\right)=L_{n+1}\left(\begin{array}{c}
L_{m+n-1} \\
L_{m-1}
\end{array}\right)+t L_{m-1}\left(\begin{array}{c}
L_{m+n-1} \\
L_{n-1}
\end{array}\right)
$$

which is a consequence of the definitions, shows that $\left(\begin{array}{c}L_{m+n} \\ L_{m}\end{array}\right)$ are indeed polynomials in $\mathbb{N}[s, t]$. Sagan and Savage in [9] call these expressions lucanomial coefficients, ${ }^{2}$ and they furnish a combinatorial interpretation for them.

One of our goals in this paper is to better understand these polynomials by analyzing their factorizations. To this end, suppose $n=p_{1}^{e_{1}} \cdots p_{r}^{e_{r}}$ is the prime factorization of $n$. We define the $n$-th flat Lucas polynomial to be the product

$$
L_{n}^{b}=L_{p_{1}} L_{p_{2}} \cdots L_{p_{r}}
$$

and the $n$-th sharp Lucas polynomial to be

$$
L_{n}^{\sharp}=\frac{L_{n}}{L_{n}^{b}} .
$$

\footnotetext{
${ }^{2} \operatorname{In}[9],\left(\begin{array}{l}L_{n} \\ L_{k}\end{array}\right)$ is denoted by $\left\{\begin{array}{l}n \\ k\end{array}\right\}$.
} 
Obviously, a flat Lucas polynomial is a polynomial. Less obvious is that a sharp Lucas polynomial is indeed a polynomial (in $s$ and $t$ ). We prove this fact in Corollary 3.10.

We define flat and sharp factorials in a conventional manner, as follows:

$$
L_{n}^{b} !=L_{n}^{b} L_{n-1}^{b} \cdots L_{1}^{b} \text { and } L_{n}^{\sharp} !=L_{n}^{\sharp} L_{n-1}^{\sharp} \cdots L_{1}^{\sharp} .
$$

Accordingly, let us introduce

$$
\left(\begin{array}{c}
L_{n} \\
L_{k}
\end{array}\right)^{b}=\frac{L_{n}^{b} !}{L_{n-k}^{b} ! L_{k}^{b} !} \text { and }\left(\begin{array}{c}
L_{n} \\
L_{k}
\end{array}\right)^{\sharp}=\frac{L_{n}^{\sharp} !}{L_{n-k}^{\sharp} ! L_{k}^{\sharp} !},
$$

and call $\left(\begin{array}{c}L_{n} \\ L_{k}\end{array}\right)^{b}$ and $\left(\begin{array}{c}L_{n} \\ L_{k}\end{array}\right)^{\sharp}$, respectively, flat and sharp lucanomial coefficients. For all $0 \leq k \leq n$, we observe the following "flat and sharp" decomposition of lucanomials:

$$
\left(\begin{array}{l}
L_{n} \\
L_{k}
\end{array}\right)=\left(\begin{array}{l}
L_{n} \\
L_{k}
\end{array}\right)^{b}\left(\begin{array}{l}
L_{n} \\
L_{k}
\end{array}\right)^{\sharp}
$$

What is really intriguing is that

Theorem 1.11. Both the flat and sharp lucanomials are polynomials in $\mathbb{N}[s, t]$.

While the proof of polynomiality of $\left(\begin{array}{c}L_{n} \\ L_{k}\end{array}\right)^{b}$ follows from a much more general fact about polynomials, when specialized to integral values of $s$ and $t$, it provided us with the following challenge.

Let $s$ and $t$ be two fixed integers. In this case, we denote the numerical sequence $\left(L_{n}^{b}(s, t)\right)_{n \in \mathbb{P}}$ by $\left(\operatorname{ev}\left(L_{n}^{b}\right)\right)_{n \in \mathbb{P}}$ in order to distinguish from the polynomials $L_{n}^{b}$. Empirical evidence suggests, for a prime number $p$, that there exists a constant $\theta=\theta_{s, t}(p) \in \mathbb{N}$ such that

$$
\nu_{p}\left(\operatorname{ev}\left(L_{n}^{b}\right) !\right)=\left\lfloor\frac{n}{\theta}\right\rfloor .
$$

We do not pursue this question here, however, the interested reader might do so. Note that when $s=2, t=-1$, the number $\operatorname{ev}\left(L_{n}^{b}\right)$ is nothing but $n^{b}$, the product of all prime numbers dividing $n$. In this case, $\theta_{2,-1}(p)=p$, and hence $\nu_{p}\left(n^{b} !\right)=\left\lfloor\frac{n}{p}\right\rfloor$.

Question: Does there exist an explicit expression for $\theta_{s, t}(p)$ ?

The second theme of our paper is on certain symmetry, which is lacking from Lucas polynomials. The specialization of $L_{n}$ at $s=x+1, t=x$ (denoted here by $D_{n}$ ) has a happy ending in the sense that 
Theorem 1.12. For all $0 \leq k \leq n$, the delannomial coefficient

$$
\left(\begin{array}{c}
D_{n} \\
D_{k}
\end{array}\right)=\frac{D_{n} D_{n-1} \cdots D_{n-k+1}}{D_{k} \cdots D_{1}} .
$$

is symmetric and unimodal in the variable $x$.

Remark 1.13. When $x=1$, the numbers $D_{n}$ evaluate to Pell numbers, which were our original motivation for the present work.

Wishing for more, we apply divided-difference calculus to Lucas polynomials and obtain various interesting corollaries, one of which we mention here. Let $\partial_{s, t}: \mathbb{N}[s, t] \rightarrow \mathbb{N}[s, t]$ denote the operator $\partial_{s, t}(F(s, t))=$ $(F(s, t)-F(t, s)) /(s-t)$. Let $\alpha \in \mathbb{N}$ and define modified Lucas polynomials by $L_{0}(s, t: \alpha)=L_{1}(s, t: \alpha)=\alpha$. For $n \geq 2$, define

$$
L_{n}(s, t: \alpha)=s L_{n-1}(s, t: \alpha)+t L_{n-2}(s, t: \alpha) .
$$

Let $S_{n}(s, t: \alpha)$ denote the divided-difference polynomial $\partial_{s, t} L_{n}(s, t: \alpha)$.

Theorem 1.14. The following hold true:

(i) $S_{n}(s, t: \alpha)=\alpha S_{n}(s, t: 1)$ for all $\alpha \in \mathbb{N}$;

(ii) $(s+t-1)$ divides $S_{n}(s, t: \alpha)$ for all $n \in \mathbb{N}$;

(iii) $\frac{S_{n}(s, t: \alpha)}{s+t-1}$ has non-negative integral coefficients, only.

An important connection between multiplicative arithmetic functions and symmetric polynomials, which we were not aware of at the time of writing this paper was pointed out to us by an anonymous referee. In the articles $[4,5,6,7]$, MacHenry and et al. develop the idea that the convolution algebra of multiplicative arithmetic functions is representable by the evaluations of certain Schur polynomials. It would be interesting to investigate our flat and sharp Lucanomials in the context of arithmetic functions in relation with symmetric functions.

We conclude our introduction with an observation on further potential interpretation of the Lucas polynomials in the context of representation theory. We plan to pursue this in the future, so we keep it brief here.

Let $q$ be a variable and $\mathbb{K}$ denote a field of characteristic zero. Consider the polynomial ring $\mathcal{P}=\mathbb{K}[q]\left[x_{1}, \ldots, x_{n}\right]$ in $n$ variables over the ring $\mathbb{K}[q]$. If $\sigma_{i}: \mathcal{P} \rightarrow \mathcal{P}, 1 \leq i<n$, denotes the $\mathbb{K}[q]$-linear operator interchanging $x_{i}$ with $x_{i+1}$, define the operators on the ring $\mathcal{P}$ by

$$
T_{i}=(q-1)\left[\frac{x_{i}-x_{i+1} \sigma_{i}}{x_{i}-x_{i+1}}\right]+\sigma_{i} \quad(1 \leq i<n) .
$$


Then the $T_{i}$ 's generate a faithful representation of a particular deformation $\mathcal{H}_{n}$ of the group ring $\mathbb{K}\left[\mathfrak{S}_{n}\right]$ of the symmetric group $\mathfrak{S}_{n}$. In fact, it is isomorphic to a specialization of the Iwahori-Hecke algebra of $\mathfrak{S}_{n}$.

Let $\rho_{(n-1,1)}$ denote the irreducible representation of $\mathcal{H}_{n}$ on the space $V$ of linear polynomials without constant terms modulo $x_{1}+\cdots+x_{n}=0$, having the polynomials $\left\{x_{n-1}+\cdots+x_{1}, \ldots, x_{2}+x_{1}, x_{1}\right\}$ as a basis. Consider the following element of $\mathcal{H}_{n}$ :

$$
H=\sum_{i=1}^{n-1}\left(T_{i}-q\right) .
$$

If $\rho_{(n-1,1)}(H)$ is the image of $H$ under the representation $\rho_{(n-1,1)}$ with respect to the above basis, then the matrix form of the image is $\rho_{(n-1,1)}(H)=$ $M_{n-1}(q)-(1+q) I_{n-1}$, where $I_{n-1}$ is the identity matrix, and $M_{n}(q)$ is the tridiagonal matrix (with super-diagonal all $q$ 's, diagonal all 0's, sub-diagonal all 1 's, and everything else 0 ). For example,

$$
\rho_{(4,1)}(H)=\left[\begin{array}{cccc}
-(1+q) & q & 0 & 0 \\
1 & -(1+q) & q & 0 \\
0 & 1 & -(1+q) & q \\
0 & 0 & 1 & -(1+q)
\end{array}\right] .
$$

Furthermore, the characteristic polynomial of $\rho_{(n-1,1)}(H)$ takes the form $C h_{n-1}(x, q)=\operatorname{det}\left[(x+1+q) I_{n-1}-M_{n-1}(q)\right]$. If we replace $q=t$ and $s=x+1+q$, then $C h_{n-1}(s, t)=\operatorname{det}\left[s I_{n-1}-M_{n-1}(t)\right]$. These determinants are easy to compute recursively by

$$
C h_{n}=s C h_{n-1}+t C h_{n-2} .
$$

Comparing initial conditions reveals a surprising connection: $C h_{n-1}(s, t)=$ $L_{n}(s, t)$, the Lucas polynomials!

\section{Preliminaries}

A closely-related family of polynomials, defined by the same recurrence $K_{n}=$ $s K_{n-1}+t K_{n-2}$ with respect to the initial conditions $K_{0}=2, K_{1}=s$ is called the family of circular Lucas polynomials. ${ }^{3}$ The ordinary and circular Lucas polynomials are interwoven by the identity:

$$
2 L_{m+n}=K_{n} L_{m}+K_{m} L_{n} \text { for all } m, n \in \mathbb{N} .
$$

\footnotetext{
${ }^{3} \operatorname{In}[9], K_{n}$ is denoted by $\langle n\rangle$.
} 
Table 1: A list of Lucas and circular Lucas polynomials

\begin{tabular}{l|l} 
Lucas polynomials & Circular Lucas Polynomials \\
\hline$L_{0}=0$ & $K_{0}=2$ \\
$L_{1}=1$ & $K_{1}=s$ \\
$L_{2}=s$ & $K_{2}=s^{2}+2 t$ \\
$L_{3}=s^{2}+t$ & $K_{3}=s^{3}+3 s t$ \\
$L_{4}=s^{3}+2 s t$ & $K_{4}=s^{4}+4 s^{2} t+2 t^{2}$ \\
$L_{5}=s^{4}+3 s^{2} t+t^{2}$ & $K_{5}=s^{5}+5 s^{3} t+5 s t^{2}$ \\
$L_{6}=s^{5}+4 s^{3} t+3 s t^{2}$ & $K_{6}=s^{6}+6 s^{4} t+9 s^{2} t^{2}+2 t^{3}$
\end{tabular}

Table 1 gives a short list of $K_{n}$ 's and $L_{n}$ 's for small $n$. Due to their recursive nature, the polynomials $K_{n}$ and $L_{n}$, as well as $\left(\begin{array}{l}L_{n} \\ L_{k}\end{array}\right)$ have nice combinatorial interpretations:

1. For all $n \geq 1$,

$$
K_{n}=\sum_{T \in \mathcal{C}_{n}} w(T),
$$

where $w(T)=s^{m} t^{d}$ such that $m$ is the number of monominos and $d$ is the number of dominos and $\mathcal{C}_{n}$ is the set of all circular tilings of a $1 \times n$ rectangle with disjoint dominos and monominos.

2. For all $n \geq 1$,

$$
L_{n}=\sum_{T \in \mathcal{L}_{n-1}} w(T)
$$

where $w(T)=s^{m} t^{d}$ such that $m$ is the number of monominos and $d$ is the number of dominos and $\mathcal{L}_{n-1}$ is the set of all linear tilings of a $1 \times(n-1)$ rectangle with disjoint dominos and monominos.

3. For a partition $\lambda$, let $\mathcal{L}_{\lambda}$ denote the set of all possible linear tilings of the rows of the Young diagram of $\lambda$, and for $\lambda \subseteq m \times n$, let $\lambda^{*}$ denote the the complimentary Young diagram of $\lambda$ in $m \times n$. Also, let $\mathcal{L}_{\lambda}^{\prime}$ denote the set of all linear tilings of the rows of $\lambda$ that do not start with a monomino. Finally, the weight $w(T)$ of an element $T=\left(T_{1}, T_{2}\right) \in \mathcal{L}_{\lambda} \times \mathcal{L}_{\mu}$ is defined as the product of the weights of the rows of $T_{1}$ and $T_{2}$. It is shown in [Theorem 3, [9]] that if $m$ and $n$ are two positive integers, then

$$
\left(\begin{array}{c}
L_{m+n} \\
L_{n}
\end{array}\right)=\sum_{\lambda \subseteq m \times n} \sum_{T \in \mathcal{L}_{\lambda} \times \mathcal{L}_{\lambda^{*}}^{\prime}} w(T) .
$$




\section{Prime divisors of Lucas polynomials}

Proposition 3.1. Let $N$ be a positive integer. Then $N$ is even if and only if $L_{2}$ divides $L_{N}$. Moreover,

$$
\frac{L_{2 N}}{L_{N}}=K_{N} \text { for any } N \geq 1 .
$$

Proof. Equation (3.2) is immediate from (2.1). When $N$ is even, the linear tiling corresponding to $L_{N}$ has length $N-1$, which is odd. Thus, each linear tiling must contain at least one monomino. The converse statement is easy to show by induction and the recurrence for $L_{N}$.

Corollary 3.3. Let $N=2^{r} n$ for some positive integers $r, n$. Then

$$
L_{N}=L_{n} \prod_{i=1}^{r} K_{\frac{N}{2^{i}}} .
$$

In particular, when $N=2^{r}$ with $r \geq 2$, we have $L_{N}=\prod_{i=1}^{r} K_{\frac{N}{2^{i}}}$.

Proof. This follows from a repeated use of Proposition 3.1.

Example 3.4. When $n=6$ :

$$
\begin{aligned}
\frac{L_{6}}{L_{3}} & =\frac{s^{5}+4 s^{3} t+3 s t^{2}}{s^{2}+t} \\
& =\frac{\left(s^{3}+3 s t\right)\left(s^{2}+t\right)}{s^{2}+t} \\
& =K_{3} .
\end{aligned}
$$

Theorem 3.5. Let $N$ be a positive integer. Then

(i) If a $\mid N$, then $L_{a} \mid L_{N}$. More precisely,

$$
\frac{L_{N}}{L_{a}}=\sum_{i=1}^{b} \frac{K_{N-i a} K_{a}^{i-1}}{2^{i}} .
$$

(ii) If $L_{a} \mid L_{N}$, then $a \mid N$.

Proof. To prove $(i)$, it suffices to prove the identity (3.6). If $a \cdot b=N$, we write

$$
\begin{aligned}
L_{N} & =L_{a+(N-a)} \\
& =\frac{K_{N-a}}{2} L_{a}+\frac{K_{a}}{2} L_{N-a} .
\end{aligned}
$$


Since $N-i a=N-(i+1) a+a$ for any $i=1, \ldots, b$, we repeatedly use $(2.1)$ in (3.7) to get:

$$
L_{N}=\sum_{i=1}^{b} \frac{K_{N-i a}}{2^{i}} L_{a} K_{a}^{i-1}
$$

For part $(\mathrm{ii})$, we already know from Proposition 3.1 that our claim is true when $a=2$, so we assume that $a>2$.

Observe that $L_{N}$ at $s=t=1$ is the $N$-th Fibonacci number $f_{N}$. Thus, if $L_{a}$ divides $L_{N}$, then $a$-th Fibonacci number $f_{a}$ divides $f_{N}$. On the other hand, it is well known that, for $n>2, f_{n} \mid f_{N}$ if and only if $n \mid N$ (see [2]). Hence, the proof is complete.

\section{Example 3.9.}

$$
L_{6}=s^{5}+4 s^{3} t+3 s t^{2}=L_{2} L_{3}\left(s^{2}+3 t\right) .
$$

Corollary 3.10. Let $N$ be a positive integer with prime factorization $N=$ $p_{1}^{e_{1}} \cdots p_{r}^{e_{r}}$, where $e_{1}, \ldots, e_{r}$ are some positive integers. Then $L_{N}$ is divisible by $\prod_{i=1}^{r} L_{p_{i}}$.

Proof. By Theorem 3.5 and induction, we rewrite $L_{N}$ in the form $L_{N}=$ $L_{p_{1}} \cdots L_{p_{r-1}} p(s, t)$ for some polynomial $p(s, t)$.

Now, if a prime factor of the polynomial $L_{p_{r}}$ divides any of $L_{p_{1}}, L_{p_{2}}, \ldots$, $L_{p_{r-1}}$, then a prime factor of the $p_{r}$-th Fibonacci number divides one of $f_{p_{1}}, \ldots, f_{p_{r-1}}$. However, it is well known that Fibonacci numbers that have a prime index do not share any common divisor greater than 1 , since [8]

$$
\operatorname{gcd}\left(f_{n}, f_{m}\right)=f_{\operatorname{gcd}(n, m)}
$$

Therefore, $L_{p_{r}}$ divides $p(s, t)$, and hence, the proof is complete.

Although $L_{2}$ divides $L_{8}$, it is not true that higher powers of $L_{2}$ divide $L_{8}$ :

$$
\frac{L_{8}}{L_{2}^{2}}=\frac{\left(s^{2}+2 t\right)\left(s^{4}+4 s^{2} t+2 t^{2}\right)}{s} .
$$

More generally, in our next result we are going to show that $L_{N}$ is not divisible by the square of any of its divisors.

Theorem 3.12. Let $p \neq 1$ be a (not necessarily prime) divisor of $N \in \mathbb{P}$. Then $L_{p}^{2}$ does not divide $L_{N}$. 
Proof. Let $n$ be such that $N=n p$. We claim that

$$
L_{N} \equiv n t^{n-1} L_{p-1}^{n-1} \quad \bmod L_{p}^{2}
$$

We show this by proving that $L_{N} / L_{p} \equiv n t^{n-1} L_{p-1}^{n-1} \bmod L_{p}$. Obviously, if $n=1$, then there is nothing to prove. To use induction, assume that our claim is true for $n$. After some cancellations, equation (1.8) implies that

$$
L_{a+b}=L_{a} L_{b+1}+t L_{a-1} L_{b} \text { for all } a, b \geq 0 .
$$

Replacing $a$ by $n p$ and $b$ by $p$ in (3.14), we have $L_{n p+p}=L_{n p} L_{p+1}+t L_{n p-1} L_{p}$. Combining this with the defining recurrence $L_{p}=s L_{p-1}+t L_{p-2}$, induction assumption and one more application of (3.14), we get:

$$
\begin{aligned}
\frac{L_{(n+1) p}}{L_{p}} & \equiv \frac{L_{n p}}{L_{p}} L_{p+1}+t L_{n p-1} \quad \bmod L_{p} \\
& \equiv n t^{n-1} L_{p-1}^{n-1}\left(s L_{p}+t L_{p-1}\right)+t L_{n p-1} \quad \bmod L_{p} \\
& \equiv n t^{n} L_{p-1}^{n}+t L_{n p-1} \quad \bmod L_{p} .
\end{aligned}
$$

Thus, it remains to show that $L_{n p-1} \equiv t^{n-1} L_{p-1}^{n} \bmod L_{p}$. We use induction on $n$ once more. If $n=1$, there is nothing to prove. Assuming validity for $n$ and using (3.14) once again, we see that

$$
L_{n p+p-1}=L_{n p} L_{p}+t L_{n p-1} L_{p-1} \equiv t^{n} L_{p-1}^{n} \quad \bmod L_{p} .
$$

Hence, we have our claim proven.

Since $L_{p-1}$ is not divisible by $L_{p}$ as $p$ and $p-1$ are relatively prime, we see that the right-hand side of (3.13) is not zero, hence $L_{N}$ is not divisible by $L_{p}^{2}$.

\section{Flat and sharp decomposition}

\subsection{Flat and sharp Lucas polynomials}

We know from Corollary 3.10 that the sharp Lucas polynomials are indeed polynomials. Due to prime involvement, finding a combinatorial interpretation for sharp polynomials is a challenging problem. Equivalently difficult is the problem of describing all monomials of a sharp (or of a flat) polynomial. Note that, if $n$ itself is a prime number, then $L_{n}^{\sharp}$ is trivial $(=1)$. More generally, suppose $n=p_{1}^{e_{1}} \cdots p_{r}^{e_{r}}$ is the prime decomposition of $n$. It is easy 
to see from the recursive definition of $L_{n}$ that $L_{n}$ is monic in $s$ with degree $n-1$ (for $n \geq 1$ ). Therefore, the $s$-degree of $L_{n}^{\sharp}$ is equal to

$$
\operatorname{deg}_{s} L_{n}^{\sharp}=n-1-\sum_{i=1}^{r}\left(p_{i}-1\right)=n-\sum_{i=1}^{r} p_{i}+r-1 .
$$

For the $t$-degree, we have

$$
\operatorname{deg}_{t} L_{n}^{\sharp}=\left\lfloor\frac{n-1}{2}\right\rfloor-\sum_{i=1}^{r}\left\lfloor\frac{p_{i}-1}{2}\right\rfloor .
$$

When $N \in \mathbb{P}$ is a power of $2, L_{N}^{\sharp}$ reveals itself rather explicitly. Indeed, we have a precise analogue of Corollary 3.3: suppose $N=2^{r} n$ for some positive integers $r, n$. Then

$$
L_{N}^{\sharp}=\frac{L_{n}^{\sharp}}{L_{2}} \prod_{i=1}^{r} K_{\frac{N}{2^{i}}} .
$$

In the special case when $N=2^{r}$ for $r \geq 2$, then

$$
L_{N}^{\sharp}=\frac{\prod_{i=1}^{r} K_{2^{i}}}{L_{2}} .
$$

Lemma 4.1. For any prime number $p$, and an arbitrary positive integer $N$, we have

$$
\operatorname{gcd}\left(L_{p}, L_{N}^{\sharp}\right)=1 .
$$

Proof. If $p$ does not divide $N$, then there is nothing to prove. So, we proceed with the assumption that $p$ divides $N$. Suppose $N=n p$ for some $n \in \mathbb{N}$, and let $g=g(s, t) \in \mathbb{N}[s, t]$ denote $\operatorname{gcd}\left(L_{p}, L_{N}^{\sharp}\right)$. Obviously, we may assume that $g$ is a non-constant polynomial. It is also evident that $g$ is a divisor of $L_{N} / L_{p}$. We know from the proof of Theorem 3.12 that $L_{N} / L_{p} \equiv n t^{n-1} L_{p-1}^{n-1}$ $\bmod L_{p}$, hence,

$$
\frac{L_{N}}{L_{p}} \equiv n t^{n-1} L_{p-1}^{n-1} \quad \bmod g .
$$

Therefore, $g$ divides the right-hand side of (4.2). In particular, specializing at $s=t=1$, we see that $g(1,1)$ divides $f_{p-1}^{n-1}$, hence, a prime factor of $g(1,1)$ divides $f_{p-1}$. But this means $f_{p}=L_{p}(1,1)$ and $f_{p-1}$ have a common prime divisor, which is absurd. Therefore $g=1$. 
Recall that $\operatorname{gcd}\left(L_{m}, L_{n}\right)=L_{\operatorname{gcd}(m, n)}$. Divisibility properties of Lucas polynomials carry over to the flattened and sharpened versions:

Theorem 4.3. Let $m$ and $n$ be two positive integers such that $m \mid n$. Then

(i) $L_{m}^{b} \mid L_{n}^{b}$ in $\mathbb{N}[s, t]$,

(ii) $L_{m}^{\sharp} \mid L_{n}^{\sharp}$ in $\mathbb{N}[s, t]$.

Proof. Part $(i)$ follows from Theorem 3.5. Part (ii) follows from part $(i)$ combined with Lemma 4.1.

\subsection{Flat and sharp lucanomials}

Theorem 4.4. For all $0 \leq k \leq n$, we have

$$
\left(\begin{array}{c}
L_{n} \\
L_{k}
\end{array}\right)=\left(\begin{array}{c}
L_{n} \\
L_{k}
\end{array}\right)^{b}\left(\begin{array}{c}
L_{n} \\
L_{k}
\end{array}\right)^{\sharp} .
$$

Proof. This is immediate from

$$
L_{n}^{\sharp !}=\frac{L_{n} !}{L_{n}^{b} !},
$$

which itself is a consequence of equation (1.10).

Clearly, the remarkable combinatorial interpretation (2.3) of $\left(\begin{array}{l}L_{n} \\ L_{k}\end{array}\right)$ exists because of polynomiality. A natural question to ask at this point is whether or not the flat/sharp lucanomials are polynomials. The answer is affirmative.

We proceed with a rather general result on "binomial coefficients" for the flattened polynomial sequences. Although we state this for polynomials only, it stays valid for sequences in an integral domain.

Theorem 4.5. Let $R$ be a polynomial algebra over a field of characteristic zero. Let $\left\{P_{n}\right\}_{n \in \mathbb{N}}$ be a sequence of polynomials from $R$ with $P_{0}=0$ and $P_{1}=1$. For each $n \in \mathbb{P}$, let $P_{n}^{b}$ denote the flattening of $P_{n}$, that is $P_{n}^{b}=$ $P_{p_{1}} \cdots P_{p_{r}}$, whenever $n=p_{1}^{e_{1}} \cdots p_{r}^{e_{r}}$ is the prime factorization of $n$. Then the associated flat binomial $\left(\begin{array}{c}P_{n} \\ p_{k}\end{array}\right)^{b}:=\frac{P_{n}^{b} \cdots P_{n-k+1}^{b}}{P_{k}^{b} \cdots P_{1}^{b}}$ is a polynomial.

Proof. If $p \in \mathbb{P}$ is a prime number, then with an abuse of terminology call $P_{p}$ "prime." We define the $P_{p}$-valuation of $P_{n}$ to be the highest exponent of $P_{p}$ in the factorization of $P_{n}$ in $R$. Since $P_{n}^{b}$ is a product of primes, the

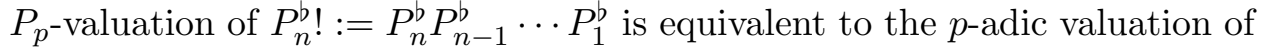
$n^{b}$ !, which is 


$$
\nu_{P_{p}}\left(P_{n}^{b} !\right)=\nu_{p}\left(n^{b} !\right)=\left\lfloor\frac{n}{p}\right\rfloor
$$

hence

$$
\nu_{P_{p}}\left(\left(\begin{array}{c}
P_{n} \\
P_{k}
\end{array}\right)^{b}\right)=\left\lfloor\frac{n}{p}\right\rfloor-\left\lfloor\frac{k}{p}\right\rfloor-\left\lfloor\frac{n-k}{p}\right\rfloor \geq 0
$$

To prove the inequality in (4.6) write $n=m p+r, k=l p+t$ where $0 \leq$ $t, s \leq p$. So,

$$
\left\lfloor\frac{n}{p}\right\rfloor-\left\lfloor\frac{k}{p}\right\rfloor-\left\lfloor\frac{n-k}{p}\right\rfloor=m-l-(m-l)-\left\lfloor\frac{r-t}{p}\right\rfloor=-\left\lfloor\frac{r-t}{p}\right\rfloor \geq 0
$$

since $r-t<p$ (possibly negative). In fact, this argument shows that $\nu_{P_{p}}\left(\left(\begin{array}{l}P_{n} \\ P_{k}\end{array}\right)^{b}\right)$ is 0 or 1 . Therefore, the a priori rational function $\left(\begin{array}{l}P_{n} \\ P_{k}\end{array}\right)^{b}$ is a polynomial.

Theorem 4.7. Both the flat and sharp lucanomials are polynomials in $\mathbb{N}[s, t]$.

Proof. Polynomiality of the flat lucanomials follows from Theorem 4.5, so, we proceed with the sharp lucanomials. Since $\left(\begin{array}{l}L_{n} \\ L_{k}\end{array}\right)$ is a polynomial, by Theorem 4.4 it is enough to show that the denominator of $\left(\begin{array}{l}L_{n} \\ L_{k}\end{array}\right)^{\sharp}$ has no divisor shared with the polynomial $\left(\begin{array}{l}L_{n} \\ L_{k}\end{array}\right)^{b}$. In light of Lemma 4.1 this is now obvious.

\subsection{Catalanomials}

In this section we extend the discussion to an $s, t$-version of the classical Catalan numbers.

Definition 4.8. A general binomial version of Catalan, the $(s, t)$-Catalan, is defined to be

$$
C_{L_{n}}:=\frac{1}{L_{n+1}}\left(\begin{array}{c}
L_{2 n} \\
L_{n}
\end{array}\right) .
$$

The flat and sharp $(s, t)$-Catalan polynomials, $C_{n}^{b}, C_{n}^{\sharp}$ are defined similarly,

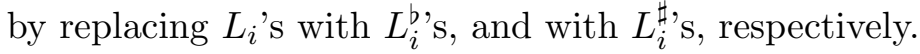

Theorem 4.9. The $(s, t)$-Catalans $C_{L_{n}}, C_{n}^{b}, C_{n}^{\sharp}$ are all polynomials in $\mathbb{N}[s, t]$. 
Proof. The first assertion is immediate from

$$
C_{L_{n}}=\left(\begin{array}{c}
L_{2 n-1} \\
L_{n-1}
\end{array}\right)+t\left(\begin{array}{c}
L_{2 n-1} \\
L_{n-2}
\end{array}\right),
$$

which is one of the properties found in [9]. The proof is completed by double induction on $n$ and $k$. For the second, it is enough to observe that $L_{n+1}^{b}$ divides $\left(\begin{array}{c}L_{2 n} \\ L_{n}\end{array}\right)$ and $\operatorname{gcd}\left(L_{n+1}^{b},\left(\begin{array}{c}L_{2 n} \\ L_{n}\end{array}\right)^{\sharp}\right)=1$ (by Lemma 4.1). The proof of the third assertion follows from that of the second.

\section{Delannomials}

Let $a, b \in \mathbb{P}$ be two positive integers. The Delannoy number $D(a, b)$ is the number of lattice paths starting at $(0,0)$ and ending at $(b, a)$ moving with unit steps $(1,0),(0,1)$, or $(1,1)$. These numbers are given by the recurrence relation

$$
D(a, b)=D(a-1, b)+D(a, b-1)+D(a-1, b-1)
$$

and the initial conditions $D(a, 0)=D(0, b)=D(0,0)=1$. The beautiful symmetry of the generating series

$$
\mathcal{D}(x, y)=\sum_{\substack{a+b \geq 1 \\ a, b \in \mathbb{N}}} D(a, b) x^{a} y^{b}=\frac{1}{1-x-y-x y}
$$

is indicative of a rich combinatorics associated with these numbers. Of particular interest is the paper [3], where Delannoy numbers find a prominent place in number theory (especially, in the discussion on the notion of the so-called local Riemann Hypothesis).

Let $x$ be a new variable, and define the polynomial $D_{n}(x), n \in \mathbb{N}$ by

$$
D_{n}(x)=\left.L_{n}\right|_{s=x+1, t=x} .
$$

It is immediate from the defining recurrence of Lucas polynomials that $D_{0}=$ $0, D_{1}=1$ and

$$
D_{n}(x)=(x+1) D_{n-1}+x D_{n-2},
$$

for $n \geq 2$. If there is no danger of confusion we remove the argument $x$ and write simply $D_{n}$ in place of $D_{n}(x)$.

The next result shows that the coefficients of $D_{n}(x)$ are the classical Delannoy numbers. 
Lemma 5.3. For each $n \geq 1$, we have

$$
D_{n}(x)=\sum_{i=1}^{n} D(n-i, i-1) x^{i-1} .
$$

Proof. Write $D_{n}=\sum_{i=0}^{n-1} d_{i}^{n} x^{i}$. Then by the recurrence (5.2) we see that

$$
\sum_{i=0}^{n-1} d_{i}^{n} x^{i}=(x+1) \sum_{i=0}^{n-2} d_{i}^{n-1} x^{i}+x \sum_{i=0}^{n-3} d_{i}^{n-2} x^{i},
$$

or equivalently, for $1 \leq i \leq n-3$,

$$
d_{i}^{n}=d_{i-1}^{n-1}+d_{i}^{n-1}+d_{i-1}^{n-2} .
$$

Assume by induction that $d_{i}^{n}=D(n-i, i-1)$. Then the recurrence (5.2) together with the induction hypothesis implies that

$$
\begin{aligned}
D(n-i, i-1)= & D((n-1)-(i-1), i-2) \\
& +D((n-1)-i, i-1)+D((n-2)-(i-1), i-2),
\end{aligned}
$$

which is equivalent to $(5.5)$.

Lemma 5.6. Preserve the notation from the proof of Lemma 5.3, and write $D_{n}=\sum_{i=0}^{n-1} d_{i}^{n} x^{i}$. Then $d_{i}^{n}=d_{n-i-1}^{n}$.

Proof. By Lemma 5.3, we know that $d_{n-i-1}^{n}=D(n-1-(n-i-1), n-i-1)$ and that $d_{i}^{n}=D(n-1-i, i)$. Obviously these are equal quantities.

Definition 5.7. The $(n, k)$-th delannomial is defined to be

$$
\left(\begin{array}{c}
D_{n} \\
D_{k}
\end{array}\right)=\frac{D_{n} D_{n-1} \cdots D_{n-k+1}}{D_{k} \cdots D_{2} D_{1}} .
$$

Let $p(x)=\sum_{i=0}^{r} a_{i} x^{i}$ be a polynomoial. If $r$ is odd, then the central monomial of $p(x)$ is defined to be $a_{j} x^{j}$, where $j=(r+1) / 2$. If $r$ is even, it is defined to be $a_{j} x^{j}$ with $j=\lfloor r / 2\rfloor$.

Theorem 5.8. For all $m$ and $n$, the delannomial $\left(\begin{array}{c}D_{m+n} \\ D_{m}\end{array}\right)$ is symmetric and unimodal.

Proof. The recurrence (1.8) induces the same recurrence on $\left(\begin{array}{c}D_{m+n} \\ D_{m}\end{array}\right)$. Since product of symmetric and unimodal polynomials is symmetric and unimodal, we only need to show that the degree of the central monomial of $D_{m+1}\left(\begin{array}{c}D_{m+n} \\ D_{m-1}\end{array}\right)$ 
matches with the central monomial of $x D_{m-1}\left(\begin{array}{c}D_{m+n} \\ D_{n-1}\end{array}\right)$. This follows from induction.

Remark 5.9. For $\left(\begin{array}{c}D_{m+n} \\ D_{m}\end{array}\right)$, there exists a combinatorial interpretation, along the lines of [9], by using dominos (weighted by $x$ ) and two kinds of monominos (weighted by $x$ and $x^{2}$ ).

\section{Divided-differences}

The Lucas polynomials bring in many interesting features, but they fail to be symmetric in the variables $s$ and $t$. For example, $L_{2}=s$. To remedy this deficit, we consider their behavior under the divided-difference operator. To be precise, we associate the sequence of polynomials defined by

$$
S_{n}(s, t)=\frac{L_{n}(s, t)-L_{n}(t, s)}{s-t} .
$$

Of course, $S_{n}(s, t)=S_{n}(t, s)$ for all $n \geq 0$. Let's record some basic properties of $S_{n}(s, t)$. The next result shows a simple algebraic relation between the two sequence of polynomials via generating functions.

Lemma 6.1. Suppose $S(x ; s, t)=\sum_{n} S_{n}(s, t) x^{n}$ and $L(x ; s, t)=$ $\sum_{n} L_{n}(s, t) x^{n}$. Then

$$
S(x ; s, t)=(1-x) L(x ; s, t) L(x ; t, s) .
$$

Proof. It is well known that $L(x ; s, t)=\frac{x}{1-s x-t x^{2}}$. Now, proceed as follows:

$$
\begin{aligned}
\frac{L(x ; s, t)-L(x ; t, s)}{s-t} & =\frac{1}{s-t}\left[\frac{x}{1-s x-t x^{2}}-\frac{x}{1-t x-s x^{2}}\right] \\
& =\frac{1}{s-t}\left[\frac{(s-t)(1-x) x^{2}}{\left(1-s x-t x^{2}\right)\left(1-t x-s x^{2}\right)}\right] .
\end{aligned}
$$

The proof is complete.

Corollary 6.2. There is a recurrence relation linking $L_{n}(s, t)$ with $S_{n}(s, t)$ :

$$
S_{n}(s, t)=s S_{n-1}(s, t)+t S_{n-2}(s, t)+L_{n-1}(s, t)-L_{n-2}(s, t) .
$$

Proof. Rewrite Lemma 6.1 in the form: $\left(1-s x-t x^{2}\right) S(x ; s, t)=(x-$ $\left.x^{2}\right) L(x, s, t)$. Taking the coefficients of $x^{n}$ on both sides of this equation reveals that

$$
S_{n}(s, t)-s S_{n-1}(s, t)-t S_{n-2}(s, t)=L_{n-1}(s, t)-L_{n-2}(s, t),
$$

which is equivalent to desired conclusion. 
The generating function for second order Fibonacci numbers, as defined in

$$
\text { http://oeis.org.A010049, }
$$

is $x(1-x) /\left(1-x-x^{2}\right)^{2}$. The next statement connects these numbers with the divided-differences $S_{n}(1,1)$.

Corollary 6.3. Let $a_{n}$ denote the specialization of $S_{n}(s, t)$ at $s=t=1$. Then

(i) $a_{n}$ is the $(n-1)$-th second order Fibonacci number;

(ii) $a_{n}=f_{n-1}+\sum_{k=0}^{n-2} f_{n-2-k} f_{k}$.

Proof. (ii) Recall that $L(x ; 1,1)=\sum_{n} f_{n} x^{n}$, where $f_{n}$ are the Fibonacci numbers. Observe also that given any $F(x)=\sum_{n} c_{n} x^{n}$, the partial sums $\sum_{k=0}^{n} c_{n}$ have generating function $\frac{F(x)}{1-x}$. From Lemma 6.1 , we have $\frac{S(x ; 1,1)}{1-x}=$ $L(x ; 1,1)^{2}$. Extract the coefficients of $x^{n}$ to obtain $\sum_{k=0}^{n} a_{k}=\sum_{k=0}^{n} f_{n-k} f_{k}$ (where Cauchy's product formula has been utilized). Since $f_{n-k}-f_{n-1-k}=$ $f_{n-2-k}$, it is easy to see that

$$
\begin{aligned}
a_{n}=\sum_{k=0}^{n} a_{k}-\sum_{k=0}^{n-1} a_{k} & =\sum_{k=0}^{n} f_{n-k} f_{k}-\sum_{k=0}^{n-1} f_{n-1-k} f_{k} \\
& =f_{n-1}+\sum_{k=0}^{n-1}\left(f_{n-k}-f_{n-1-k}\right) f_{k} \\
& =f_{n-1}+\sum_{k=0}^{n-2} f_{n-2-k} f_{k} .
\end{aligned}
$$

To get $(i)$, use $S(x ; 1,1)=(1-x) L(x ; 1,1)^{2}=x\left[\frac{x(1-x)}{\left(1-x-x^{2}\right)^{2}}\right]$. The proof follows.

In the next result we obtain a recurrence for the divided-difference $S_{n}(s, t)$.

Corollary 6.4. Preserve the notations from Lemma 6.1. Write $S_{n}$ for $S_{n}(s, t)$. For $n \geq 4$, we have

$$
S_{n}=(s+t) S_{n-1}+(s+t-s t) S_{n-2}-\left(s^{2}+t^{2}\right) S_{n-3}-s t S_{n-4} .
$$

Proof. Once more, Lemma 6.1 implies $\left(1-s x-t x^{2}\right)\left(1-t x-s t^{2}\right) S(x ; s, t)=$ $x^{2}-x^{3}$. Equivalently,

$$
\left[1-(s+t) x-(s+t-s t) x^{2}+\left(s^{2}+t^{2}\right) x^{3}+s t x^{4}\right] S(x ; s ; t)=x^{2}-x^{3} .
$$


Now, simply compare the coefficients of $x^{n}$ on both sides of the last equation.

Here is an amusing corollary with beautiful symmetry.

Corollary 6.5. For $s, t \in \mathbb{P}$, we have the numerical series evaluation

$$
\sum_{n \geq 0} \frac{S_{n}(s, t)}{(s+t)^{n+1}}=\frac{1}{s t(s+t-1)} .
$$

Proof. Corollary 2.6 of [1] states that

$$
\sum_{n} \frac{L_{n}(s, t)}{(s+t)^{n+1}}=\frac{1}{t(s+t-1)}
$$

Thus,

$$
\begin{aligned}
\sum \frac{S_{n}(s, t)}{(s+t)^{n+1}} & =\sum \frac{L_{n}(s, t)-L_{n}(t, s)}{(s-t)(s+t)^{n+1}} \\
& =\frac{1}{s-t}\left[\frac{1}{t(s+t-1)}-\frac{1}{s(s+t-1)}\right]=\frac{1}{s t(s+t-1)}
\end{aligned}
$$

Remark 6.6. Despite the above plethora of facts, one aspect of the symmetric functions $S_{n}(s, t)$ remains undesirable from a combinatorial viewpoint: the coefficients are not all non-negative. Fortunately, all is not lost because there is a quick fix as will be seen below.

Let $\alpha \in \mathbb{N}$. While maintaining the recursive relation for Lucas polynomials, we make a slight alteration to the initial conditions: assume $L_{0}(s, t$ : $\alpha)=L_{1}(s, t: \alpha)=\alpha$. For $n \geq 2$, define

$$
L_{n}(s, t: \alpha)=s L_{n-1}(s, t: \alpha)+t L_{n-2}(s, t: \alpha) .
$$

Let $S_{n}(s, t: \alpha)$ denote the divided-difference polynomial that is associated with $L_{n}(s, t: \alpha)$.

Theorem 6.7. The following hold true:

(i) $S_{n}(s, t: \alpha)=\alpha S_{n}(s, t: 1)$ for all $\alpha \in \mathbb{N}$;

(ii) $(s+t-1)$ divides $S_{n}(s, t: \alpha)$ for all $n \in \mathbb{N}$;

(iii) $\frac{S_{n}(s, t: \alpha)}{s+t-1}$ has non-negative integral coefficients, only. 
Proof. ( $i)$ The defining recurrence and initial conditions imply the homogeneity $L_{n}(s, t: \alpha)=\alpha L_{n}(s, t: 1)$. From here, it is evident that $S_{n}(s, t: \alpha)$ inherits the same property. Routine standard methods give

$$
L(s, t: 1):=\sum_{n} L_{n}(s, t: 1) x^{n}=\frac{1-(s-1) x}{1-s x-t x^{2}} .
$$

One can easily verify that $\sum_{n} S_{n}(s, t: 1) x^{n}=\frac{(s+t-1) x^{3}}{\left(1-s x-t x^{2}\right)\left(1-t x-s x^{2}\right)}$. In particular,

$$
\sum_{n} \frac{S_{n}(s, t: 1)}{s+t-1} x^{n}=\frac{x^{3}}{\left(1-s x-t x^{2}\right)\left(1-t x-s x^{2}\right)}=x L(s, t) L(t, s)
$$

simultaneously demonstrates the divisibility in $(i i)$ as well as the claim in (iii).

Remark 6.8. It is worthwhile to note that $L_{n}(s, t: 1)=L_{n}(s, t)+t L_{n-1}(s, t)$. As a result, the modified Lucas polynomials also retain a combinatorial interpretation much as the ordinary ones. Such as simultaneous tiling of a pair of rectangles, one $1 \times(n-1)$ and the other $1 \times n$, where the latter always begins with a domino.

\section{Acknowledgements}

The second and the third authors are partially supported by a Louisiana Board of Regents Research and Development Grant 549941C1. Authors thank the anonymous referee for her/his comments and pointers on the literature.

\section{References}

[1] T. Amdeberhan, X. Chen, V. Moll, and B. Sagan, Generalized Fibonacci polynomials and Fibonomial coefficients. To appear in Annals of Comb. MR3282645

[2] M. Bicknell and V. Hoggatt Jr., A primer for the Fibonacci numbers: Part IX. The Fibonacci Quarterly 9 (December 1971), no. 5.

[3] D. Bump, K. Choi, P. Kurlberg and J. Vaaler, A local Riemann hypothesis, I. Math Z. 233 (2000), 1-19. MR1738342 
[4] T. MacHenry, Generalized Fibonacci and Lucas polynomials and multiplicative arithmetic functions. Fibonacci Quarterly 38 (2000), 17-24. MR1760472

[5] T. MacHenry and G. Todose, Reflections on isobaric polynomials and arithmetic functions. Rocky Mountain J. Math. 35 (2005), no. 3, 901928. MR2150315

[6] T. MacHenry and K. Wong, A correspondence between the isobaric ring and multiplicative arithmetic functions. Rocky Mountain J. Math. 42 (2012), no. 4, 1247-1290. MR2981044

[7] H. Li and T. MacHenry, The convolution ring of arithmetic functions and symmetric polynomials. Rocky Mountain J. Math. 43 (2013), no. 4, 1227-1259. MR3105320

[8] P. Ribenboim, My numbers, my friends. Springer-Verlag, New York, 2000. MR1761897

[9] B. Sagan and C. Savage, Combinatorial interpretations of binomial coefficient analogs related to Lucas sequences. Integers 10 (2010), A52, 697-703. MR2797767

Tewodros Amdeberhan

Department of Mathematics

Tulane UNIVERSITY

New ORLEANS

USA

E-mail address: tamdeber@tulane.edu

Mahir Bilen Can

Department of Mathematics

Tulane University

New ORLEANS

USA

E-mail address: mcan@tulane.edu

Melanie Jensen

Department of Mathematics

Tulane University

New ORLEANS

USA

E-mail address: mjensen1@tulane.edu

RECEIVED 12 June 2014 